\title{
WILHELM VON SCHOLZ
}

\author{
Prof. Dr. Ernst Alker \\ Universidade de Friburgo - Suíça
}

\section{Ansprache aus Anlass seines fünfundachtzigsten Geburtstages}

Zu den Eigentümlichkeiten unserer Zeit gehört es, dass die Stellung des Dichters in jener Form der sozialen Symbiose, die man Nation nennt, problematisch geworden ist.

Worin besteht die Aufgabe des Dichters, könnte man fragen. Ist er lediglich ein Schöpfer von Werken, deren höhere Heiterkeit den Gebildeten eine geschmackvolle Entspannung bietet? Ist er Gestalter des schönen oder hässlichen Widerscheins des Lebens? Ist er ein Mahner, der den Menschen einen Spiegel ihrer Unvollkommenheiten und Gebresten vorhält? Ist er ein Propagandist gehobener Art, der die Absichten einer Ștaatsführung durch das Mittel des Wortkunstwerkes plausibel machen will? Ist er ein Leidender, einer von jenen, die bekennen müssen und die ihre Schuld - und Angstgefühle so formulieren, dass das Private zum Überprivaten wird im Sinne berühmter Verse Henrik Ibsens: "Leben heisst dunkler Gewalten Spuk bekämpfen in sich, Dichten Gerichtstag halten über das eigene Ich"? Ist er ein homo ludens - der grosse niederländische Kultuhistoriker Huizinga hat den Terminus geschaffen - , der den genialen Spieltrieb des Kindes in das geheimnisvolle Reich der Kunst rettet?

Ob man den erwähnten Definitionen vom Wesen des Dichters zustimmt oder anderen Deutungsmöglichkeiten, in keinem Fall wird er - insonderheit, wenn ihm das Wortkunstwerk den letzthinnigen Lebenssinn bedeutet - ausschliesslich Träger nur einer Erscheinungsform des poeta sein können. In der Gesellschaft der Gegenwart, voll von ausgeprägten soziologischen Kategorien, nimmt er eine ebenso merkwürdige wie unsichere Stellung ein. Am besten belegt dies die Tatsache, dass zwar der Schriftsteller (dessen Gewerbe oft der Bemühung des Dichters sehr nahesteht 
und doch nie vollkommen mit seinem Beruf identisch ist) eine anerkannte soziologische Kategorie vergegenwärtigt (verwendbar für Adress - und Fernsprechverzeichnisse, zulässig bei Melde und Finanzämtern), nicht aber, im Gegensatz zur Position des bildenden Künstlers oder Komponisten, der Dichter. Eine Visitenkarte, die den Titel "Dichter" unter den Vor — und Familiennamen setzt, dürfte Gelächter oder Entrüstung auslösen; der durch sie Vorgestellte würde Achselzucken oder Missfallen erregen namentlich dann, wenn der Poet sich in schwierigen wirtschaftlichen Verhältnissen befindet: es geschieht nicht selten, dass einem vermögenslosen, von keinem Mäzen unterstützten Dichter ohne materielle Sicherung durch den Ertrag seiner Publikationen vom Wohlfahrtsamt der wohlmeinende Rat erteilt wird, sich einen "richtigen" Beruf zu suchen, etwa Gebrauchslyrik für die Propaganda einer Zigarettenmarke zu erstellen, Tatsachenberichte für Illustrierte zu schreiben, Texter bei der Filmindustrie zu werden.

Die Bedeutung eines Dichters für sein Volk und für eine Epoche im Dasein einer Nation ist aus den angegebenen Gründen gerade in unseren Tagen nur selten deutlich erkennbar und wird meistens erst nach einem mühevollen Leben im Dienst des Wortes und des Geistes evident - dann, wenn es deutlich geworden ist, dass sein Schaffen das Gedächtnis der Nation (das ist die Summe alles werthaften Schrifttums in seiner Sprache) vermehrt hat.

Wenn die Erkenntnis (oder wenigstens Ahnung) der Bedeutung eines Dichters sich durchsetzt, ist dies für ihn und sein Werk die schönste Belohnung - für ein Lebenswerk, das mit einem Monolog begann, zum Dialog mit Seelenverwandten wurde und schliesslich überpersönliche Resonanz zeitigte. Nicht allen Dichtern ist es vergönnt, von der ersten zur zweiten Stufe der Wirkung und von dort zur Erfüllung ihrer Sendung aufzusteigen.

Wilhelm von Scholz, dessen fünfundachtzigsten Geburtstag wir feiern, hat diese Stadien der kontrollierbaren dichterischen Wirkung durchlaufen und darf am Abend eines langen und angestrengten Lebenstages den Anspruch erheben, zu den repräsentativen Gestalten des deutschen Geistes der Gegenwart gezählt zu werden. 
Denn seine Dichtung vergegenwärtigt - ohne dass dies je bewusste Intention gewesen wäre - zuvörderst eine intensive Verschmelzung des Westlichen und des Oestlichen. Bedeutungsvoll ist dies in einer Zeit, wo es nicht eine deutsche Literatur gibt, sondern wenigstens zwei deutsche Literaturen, die westdeutsche und die der DDR (Literaturen, welche in sprachlicher Hinsicht bereits unverkennbare Trennungstendenzen zu zeigen beginnen).

Die Verschmelzung des Westlichen und Oestlichen ist so intensiv, dass als Nebeneffekt sich ein Meinungsstreit darüber entwickelt hat, ob Wilhelm von Scholz ein schlesischer oder ein westdeutscher Dichter wäre. Die Vertreter der verschiedenen Auffassungen argumentieren mit Hinweisen, die in jedem Fall sehr berechtigt sind und doch nicht die eindeutige Entweder-OderEntscheidung bewirken können. In Wilhelm von Scholzens Büchern vollzog sich von Anfang an (also bereits in einer Zeit, als eine Problemlage wie die angedeutete vollkommen wirklichkeitsfern zu sein schien) die Synthese zweier regional bestimmter Weltbilder, deren Kontraste keinem Betrachter zu entgehen vermögen. Die neustämmische schlesische Literatur verfügt meistens infolge ihrer besonderen siedlungsgeschichtlichen Voraussetzungen nicht über jene Sicherheit und Weihe der Form, welche dem westdeutschen Schrifttum in so hohem Masse eignet, das von einer mehr als tausendjährigen Kulturtradition genährt wird. Das Wesen der sich immer wieder leidenschaftlich der Chaotik der Entgrenzungen und Tiefendimensionen hingebenden ostdeutschen und damit auch der schlesischen Dichtung steht in einem naturgegebenen Gegensatz zum Formwillen und zu der Formtradition des deutschen Westens, für den Vollendung der künstlerischen Aussage mehr bedeutet als die in der dichterischen Weisung sich offenbarende Unendlichkeit der Horizonte. Wilhelm von Scholz ist mit instinktiver Sicherheit einen Weg gegangen, der infolge seines genetisch bedingten Wesensgefüges doppelt bedroht und gefährlich war: durch die östliche Versuchung zu dunkler, gestalt- und formloser Orphik, durch die westliche Versuchung zu einer auf das Nur-Ornamentale und Undämonische sich beschränkenden zeremoniösen Zelebrierung der Form. Wilhelm von Scholz hat das bereits in seinem Fami- 
liennamen zum Ausdruck kommende schlesische Ahnenerbe trotz der jahrzehntelangen Beheimatung am Bodensee bewahrt, nicht minder aber alle Segnungen dieser reichen Kulturlandschaft zu einem Besitz gemacht, der das Gesamt seines Schaffens prägt und durchglüht.

Diese Synthese des Westlichen und Oestlichen zeigt sich auch auf unverkennbare Weise im stofflichen Bereich. Der deutlich wahrnehmbaren Neigung des Dichters zur Bevorzugung von Motiven und Lokalen okzidentaler Herkunft, zur Aufnahme und Gestaltung von Anregungen, die aus dem deutschen Südwesten, aber auch aus Frankreich, Italien und Spanien kommen, steht eine in materialmässiger Hinsicht sehr sparsame Frequenz schlesischer Fermente gegenüber. Anderseits hat eine Leistung zentraler Art, der grosse Meisterroman "Der Weg nach llok" in intensivster Weise das Breslau des fünfzehnten Jahrhunderts zur Spielbühne und spiegelt mehr als jeder sonstige prosaepische Versuch die abgründigen seelischen Erregungen im Herbst des schlesischen Spätmittelalters. Trotz der Tatsache, dass sonst schlesischer Raum nur ausnahmsweise für Werke Wilhelm von Scholzens in Frage gekommen ist, zeigt sich nicht selten eine unverkennbare wechselseitige Durchdringung des von Sehnsucht nach dem Unbestimmten und Dunklen erfüllten Östlich-Schlesischen und des nach sicherem Kontur strebenden Westlich-Süddeutschen, jenes Westlichen, dem man seinen uralten Bezug zur spätantiken Welt und zu ihren Jahrhunderte hindurch wirksamen Ausstrahlungen anmerkt.

In diesem Zusammenhang sei auf ein Werk Wilhelm von Scholzens hingewiesen, das in seinem tiefsten Wesen Affinität zu einer typisch schlesischen Leistung bewährt. Des Dichters graziöse, von höchstem Ernst bis zur ausgelassensten Komik schwingende Komödie "Vertauschte Seelen" vollzieht sich in einem fabulösen asiatischen Königreich Mousel an einem Fürstenhof, in zeitlosem orientalischen Kostüm und mit leuchtendem Dekor; Hermann Stehrs Meisternovelle "Der Schimmer des Assistenten" - die unheimliche Durchleuchtung eines scheinbar nur psychopathologischen Vorfalls - spielt in einem mit düsterster Sachlichkeit geschilderten und überaus trostlosen Industrieort 
des Waldenburger Kohlenreviers; es wird berichtet, wie ein lebensbanger und unvitaler Büroassistent der Verwaltung der "Gottessegen-Grube" durch einen für ihn unlösbaren Konflikt aus seiner Seele in eine andere flüchtet. Trotz der ungeheuren Distanz in Hinsicht von Ort und Zeit, von Diktion, Sprache und Stil manifestiert sich in beiden Werken die gemeinsame; die sehr schlesische Wurzel.

Die ausgeprägte weistöstliche Komponente ist bloss einer der vielen Wesenszüge in der reich profilierten Persönlichkeit Wilhelm von Scholzens. Denn er hat - das sei besonders hervorgehoben - in seinen Wortkunstwerken die Schatten sich ankündigender unerhörter Veränderungen und Vollzüge vorweggenommen. In seinem Sensorium vergegenwärtigt sich eine Art vorahnender Seismographie für die gigantischen Erschütterungen des neuzeitlichen Weltbildes durch die Problematisierung der klassischen Physik. Schon im frühen lyrischen Bemühen war ein neues Raum- und Zeitgefühl vorhanden gewesen. Die infinitesimale Musik des Weltraums klingt, mag sein, unbestimmt und zögernd, aber vernehmlich in Versen, für welche Zeit und Raum auswechselbare, sich gegenseitig bedingende Mächte sind. Grosse Denker haben Jahrzehnte später das exakt formuliert, was Wilhelm von Scholz dank dichterischer Intuition gespürt und gleichsam als vages ins traumbeladene Wort geborgen hat.

Indessen, Wilhelm von Scholz kann nicht nur den Anspruch erheben, Vorahner dessen zu sein, was man heute als Weltgefühl der Epoche der Relativitätstheorie und der Expansion ins Weltall bezeichnen könnte, er hat nicht minder die Fähigkeit zur Deutung mysteriöser Chiffren einer im Menschen wesenden Uber- und Unterwelt erwiesen. Für des Dichters Empfinden bilden Gegenwart, Vergangenheit und Zukunft eine Einheit. Dadurch ist er empfänglich für das, was man mit unzulänglichen Ausdrücken "okkultistisch" oder "parapsychologisch" nennt, für das Einwirken einer jenseits der Sinneserfahrungen wirkenden seelischen Dimension. Sie offenbart sich in Ahnungen, in Wahrund Wachträumen, in geheimnisvollen Vorankündigungen kommender Dinge, in Duplizitäten der Ereignisse, in bestürzenden Zufällen, in Vorformen herannahenden Schicksals. In vielen 
der Verse des Dichters, in seinen Dramen und in seiner Prosaepik ertönen Akkorde aus einem Grenzgebiet des Dies- und Jenseitigen, aus einem transrationalen Zwischenreich. (Eine Sammlung seiner Novellen trägt den repräsentativen Titel "Zwischenreich".) Uber- und Unterwelt stossen in den Alltag hinein. Das gemeisterte und traumbeladene dichterische Wort (voll von jener Hintergründigkeit, die an sich im Urphänomen des Wortes verkapselt ist) bewirkt ohne Verzicht auf logische Abläufe und Grammatik das dunkle Kolorit der Gestalten und Geschehnisse, ihre Tiefe und Magie. Das gemeisterte dichterische Wort adelt ferner Stoffe, die bedroht sind vom Absacken in spiritistische Gruselmären und verworrene Moritaten. Die Deutung des Mysteriösen ergibt sich nicht aus beigefügten Erläuterungen, sondern aus dem Bericht und aus der Gestaltung.

Diese Besonderheit der Kunst Wilhelm von Scholzens wird in einleuchtender Weise wirkungsmächtig in den grossen Romanen "Perpetua", "Der Weg nach llok" und "Unrecht der Liebe" sowie in einer Reihe von Novellen.

Was den Roman "Unrecht der Liebe" angeht (ein vielleicht auch von den Verehrern der Kunst des Dichters unterschätztes Werk), so offenbart sich hier ein weiteres Phänomen. Die Grundmelodie der Geschichte eines Richters, der seine Frau mordete und das Verbrechen durch die ihm zur Verfügung stehenden Machtmittel verhehlen kann, ist das Walten der rächenden Nemesis: die Geopferte reisst den superklugen Täter in den Abgrund des Todes. Es ist erstaunlich, wie sehr die zentrale Stimmung des Buches übereinstimmt mit der eines (aus mancherlei Gründen fast ganz unbeachtet gebliebenen) Werkes des grossen schwedischen Botanikers Carl von Linné. In unerhörtem Gegensatz zu seinem allbekannten zeitgemässen und offiziellen Weltbild, das vom Glauben an die Mission des aufklärerischen Rationalismus erfüllt war, sammelte Linné in höchst privaten und streng gehüteten Aufzeichnungen alle ihm bekannt gewordenen Fälle vom unerbittlichen und bisweilen in merkwürdigster Weise sich vollziehenden Wirken der Nemesis.

Die Übereinstimmung der Seelenlage jenes Linné, der die "Nemesis"-Notizen niederschrieb, und der des Dichters Scholz 
ist nicht etwa Produkt eines banalen stofflichen Kontakts, sondern etwas Essentielles und deshalb Entscheidendes. Denn Wilhelm von Scholz hat eine Schrift veröffentlicht (sie ist der Höhepunkt seiner reichen Essayistik), welche, gestützt auf Berichte über selbst oder von anderen erfahrene Vorfälle, die These vom Zufall als einer Vorform des Schicksals verficht. Ebenso wie die "Nemesis"-Aufzeichnungen Linnés - die Scholz nicht gekannt haben kann - hebt sich aus dem Buch mit dem Titel "Der Zufall und das Schicksal" ein gespenstisches, von Dämonen und Lemuren bevölkertes theatrum mundi, dessen Schlüsselwörter die Substantiva: Vor-fall, Zu-fall, Schick-sal, Ce-schick sind. Einige Sätze aus dem Buch können als gleichnishaft gelten für das Weltgefühl des Dichters und die von ihm erfühlte Lebensdeutung: "Ich bin überzeugt, dass man... den Weg zur Erkennung der warnenden und bejahenden Vorzeichen finden wird. Vielleicht entstehen die Vorzeichen durch eine Art Ausstrahlung grösseren Geschehens, das im kleineren, rascheren Geschehen sich selbst als Schatten schon vorausläuft; vielleicht sind sie wie die ganz feine erste Schneeflocke, die, nur von ganz aufmerksamen Augen beachtet, meist den beginnenden Schneefall anzukündigen pflegt." Eine zum Mystischen tendierende Tiefenschau spricht aus folgenden Ausführungen des Dichters: "Der alte Gedanke, dass das Leben ein Traum sei, verdeutlicht sich in diesem Zusammenhang auch zu der dichterischen Erkenntnis: nicht ein Traum, den wir träumen, sondern einer, in dem wir geträumt werden. Mich hat wohl nie die gedankliche Folge einer Entdeckung, einer Beobachtung, die Bestätigung auch ohne Weg bisher erahnter Schlüsse so bewegt wie diese Vorstellung, dass Erde und Leben, dass dieses ganze uns bedrückende Geschehen nichts als das Innenleben eines umfassenden Geistes sein kann, der vielleicht für seine körperlosen Vorstellungen hält, was uns volle und bittere Wirklichkeit dünkt; dass, wo wir Schicksale, merkwürdige Zufälle, Fügungen und was sonst sehen, nur dasselbe Assoziationsgesetz wirkt wie in unserer Seele; dass das Gesetz unseres psychischen und des äusseren Geschehens vielleicht dasselbe ist!"

In meinen bisherigen Ausführungen habe ich mich bemüht, 
einige Wesenszüge zu verdeutlichen, die für die Dichterpersönlicnkeit Wilhelm von Scholzens entschaidend sein dürften. Diese Materialien für eine Bildnisskizze seien nun in einer Rückschau zusammengefasst.

Der soziologische Nenner der Position des Dichters Wilhelm von Scholz ist gewährleistet durch die in ihm sich manifestierende Ambivalenz des Schlesischen und des Südwestdeutschen, die er immer wieder zu einer höheren Einheit verschmolzen hat. Im Laufe eines langen und durch schöpferische Tätigkeit im Reich des Ceistes gesegneten Lebens ist er - das wird in der Epoche des Kampfes um die Wiedervereinigung der beiden Deutschland evident - ein Zeuge für die Einheit Deutschlands, zwar nicht als Kombattant in den politischen Auseinandersetzungen, sondern durch die Tatsache seiner Existenz und seines Werkes. Dies zeigt, dass in seinem Fall die Stellung des Dichters in der Symbiose unangefochten ist von der sonst sich sehr geltend machenden zeitbedingten Problematik der gesellschaftlichen Funktion des dichterischen Menschen, dies zeigt auch, dass er ein guter Bürger war und ist in der Welt seiner erlebten Mitzeit (von der werthafte autobiographische Bücher berichten) und unserer Gegenwart.

Solche Behaustheit in unserer Gegenwart (die keineswegs im Geistigen eine so kärgliche Epoche sein dürfte, wie oft behauptet wird) schliesst nicht aus, dass Scholz letzthin über der Zeit steht, dass seinem Schaffen ein prophetischer Zug eignet, dass er sich als aufgeschlossen erwiesen hat für das Kommende, für die Konsequenzen der gewaltigen Wandlungen unseres physikalischen Weltbildes. In seinen Werken sind Ahnungen von der Veränderung der Position des Menschen im Kosmos vorhanden (bewirkt durch die Entdeckung der Identität der Begriffe Raum und Zeit).

Sein dichterisches Wort erhellt die Tiefe der Innenwelt, ihr Oben und Unten, die Zusammenhänge von Schicksal und Zufall und das mysteriöse Walten der Nemesis (das, dies sei nebenbei bemerkt, geheime Zentrum aller dramatischer Kunst seit dem Beginn der Neuzeit). Dunkle Gewalten werden in das Befreiende exakter Gestaltung gehoben, durch das beschwörende dichterische 
Wort kann (um im Gleichnis zu sprechen) Eurydike aus der Däm.. merwelt des Hades emporsteigen.

Wilhelm von Scholz hat - ohne dass ihm dies irgendwie Programm gewesen wäre - die Situation des Deutschen und damit bis zu einem gewissen Grade auch des Europäers zu durchleuchten vermocht. Er hat dies getan durch das Mittel des schöpferischen Wortes und durch die dichterische Weisung: das heisst, dass er nicht dem Wort seine Erkenntnisse aufzwang, dass er sich vom Worte, von der Sprache tragen liess wie der gute Schwimmer, der mit instinktiver Sicherheit Eigenbewegung und Kraft des strömenden Elements zu harmonisieren versteht.

Die Kunst Wilhelm von Scholzens war von mancherlei Gefährdungen umwittert, insonderheit von der Gefährdung des SichVerlierens, von der Gefährdung der Politisierung, von der Gefährdung durch den Obskurantismus, von der Gefährdung der Gewaltsamkeit dort, wo nur Horchbereitschaft und Demut gelten darf.

Aber weil er solchen Gefährdungen nie ausgewichen ist, sondern sie überwunden, weil er die Probe bestanden hat, gelang ihm die Gestaltung des Widerscheins der Welt und der Tiefendimension des Menschlichen und des Aussermenschlichen. Er konnte vorstossen in jene geheimnisvollen Bereiche, wo der Mensch, ausgesetzt kosmischen Gewalten, den Sternen verfällt, wo er zum Hauch im All wird. Zugang wurde erschlossen zum Makrokosmos, aber auch zum Mikrokosmos, wo in den Tiefen der Seele Schicksal wächst des Einzelnen und des Volkes.

In vier ad se ipsum gerichteten Verszeilen, hat der Dichter einst, in der Mitte des Lebens, das Bild seines Weges entworfen, den er als "Wandernder" ging und gehen wird:

"Zeitlose Ewigkeit war einst dein Traum.

Längst wurdest du Zeit und wanderst durch den Raum.

Du weisst, dein Weg ist nur ein kurzes Stück, dein Ziel der Abend. Doch dein Schritt ist Glück." 